Janine Spethmann, Kathrin Schlüter and Kathrin Schlatterer*

\title{
Laboratory medicine contributions to patient blood management concepts
}

https://doi.org/10.1515/labmed-2017-0148

Received December 21, 2017; accepted April 5, 2018; previously published online May 12, 2018

\section{Abstract}

Background: A review of the literature shows that the role of laboratory diagnostics in the multidisciplinary concept of patient blood management (PBM) is underrepresented, so the contribution that laboratory diagnostics can make needs to be elucidated.

Methods: The central objective of this study was to decrease the volume of blood drawn for laboratory diagnostics as part of a hospital quality management project focussed on increasing efficiency and reducing the turnaround time (TAT) of laboratory results. Optimization potential within the complete preanalytical process was reviewed to obtain potential improvement measures. These measures fell into two groups: training to reduce preanalytical errors in the entire hospital and using blood collection tubes with lower nominal fill volumes.

Results: A validation of the success of these measures was performed after 1 year. The occurrence of several blood collection non-compliances had decreased substantially. By changing the blood collection tube volume, approximately 177 L less patient blood was drawn for diagnostic purposes in the 384-bed hospital per year. For geriatric and intensive care unit (ICU) patients, there was a very significant reduction in diagnostic blood loss $(-27.2 \%$ ICU, $-42.5 \%$ geriatric patients). The number of tubes used per patient per day decreased by $13 \%$.

Conclusions: In conclusion, this approach represents an important lab-side contribution to PBM concepts.

Keywords: patient blood management; preanalytical sample quality; reduction of diagnostic blood loss.

\footnotetext{
*Correspondence: Kathrin Schlatterer, Institut für Laboratoriumsmedizin, Sankt Gertrauden-Krankenhaus, Paretzer Str. 12, 10713 Berlin, Germany,

E-Mail: kathrin.schlatterer-krauter@sankt-gertrauden.de; and University Medicine of Greifswald, Institute of Clinical Chemistry and Laboratory Medicine, Greifswald, Germany Janine Spethmann: Sankt Gertrauden-Krankenhaus, Berlin, Germany Kathrin Schlüter: BD Life Sciences, Heidelberg, Germany
}

\section{Introduction}

Due to medical, social and economic changes, there is a growing scarcity of donated blood, as well as awareness and evaluation of immunomodulation by allogeneic blood transfusions (TRIM). This has led to different approaches to reduce the number of blood transfusions. One of these approaches is the multidisciplinary patient blood management (PBM) concept, which has been promoted by the World Health Organization (WHO) since 2011 [1]. Presurgical examinations for the detection and adequate treatment of anemia, as well as an efficient surgical coagulation management with the goal of reducing the need for intra- and postsurgical erythrocyte transfusions are key areas.

However, the volume of blood collected for laboratory diagnostics also contributes to a patient's total blood loss. Blood tests, necessary, more frequently and for a longer time period necessary in severely ill patients, are capable of inducing iatrogenic anemia leading to a need for a blood transfusion [2]. The use of blood collection tubes with a lower nominal volume has been suggested as part of PBM to help avoid iatrogenic blood loss (https//www.patientbloodmanagement.de/pbm-massnahmenbuendel/). An institution's blood collection strategy should be regularly subjected to critical reviews as, due to analytical methodological developments, blood volumes needed for blood tests have decreased steadily over a number of decades [3]. If, as a result of these reviews, changes are necessary, appropriate preanalytical improvements should be conducted in parallel, potentially leading to improvements in sample quality and therefore to a decrease in repeated blood collections and hence an improvement in laboratory analysis turnaround time (TAT).

The overall aim of this study was to make a laboratory contribution to PBM efforts accompanied by communication of PBM concepts to hospital staff in order to increase awareness of the impact of diagnostic blood loss.

Following the quality assurance plan-do-check-act (PDCA) cycle, a project plan was established. First, the preanalytical process was reviewed hospitalwide in order to identify opportunities for improvement. A preanalytical phase audit revealed several non-compliances and sample quality issues. This analysis led to a multimodal, targeted approach to achieve an optimization of preanalytical 


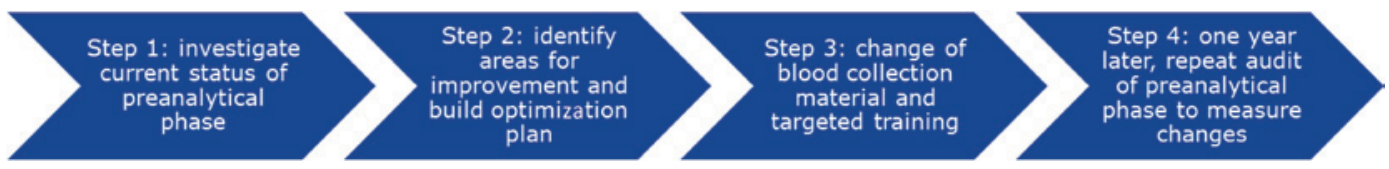

Figure 1: Steps of the targeted optimization strategy.

sample quality combined with a blood-saving collection strategy (Figure 1), enabling an improvement in TAT and an increase in hospital efficiency. One year after the implementation of the measures, the audit was repeated in order to verify and, where possible, quantify changes (Figure 1). Saving of blood by reducing blood collection and for geriatric patients with a stay over a period of 1 week tube volumes was calculated for patients with a stay of at least 7 days in the intensive care unit (ICU) over a period of 4 weeks and for geriatric patients over a time period of 1 week.

\section{Materials and methods}

\section{Reduction of blood volume withdrawn for laboratory diagnostic purposes}

A critical review of the tubes used in the hospital was performed, followed by an assessment of the blood volumes currently needed for laboratory diagnostics following ongoing analytical and methodological developments. For this purpose, the complete analytical test profile of the laboratory was evaluated with respect to minimizing sample volumes. Tubes with lower nominal filling volumes [with the exception of ethylenediaminetetraacetic acid (EDTA) tubes for hematology and immune hematology analysis (Table 1)] were introduced as part of the process of changing from a Monovette to a Vacutainer system. For geriatric patients, hematology EDTA tube collection volumes were also reduced due to the likelihood of difficult venipunctures, whereas for all other patients there was an increase from 2.7 to $3.0 \mathrm{~mL}$. The increase in EDTA immune hematology tube volumes is because for the Monovette system two tubes, each $4.9 \mathrm{~mL}$, were used for blood group analysis and crossmatching of erythrocyte concentrates. This was reduced to one $6.0 \mathrm{~mL}$ tube in tandem with the introduction of an additional verification of the blood group in the crossmatch process in order to maintain a high level of patient safety by avoiding patient misidentification. In addition, non-gel clinical chemistry tubes were changed to gel separator tubes, leading to a higher sample yield and prolonged analyte stability. This higher yield and improved stability should reduce the need for repeated blood collection procedures, thereby affording a further saving of blood within diagnostic processes.

In order to quantify the final saving of blood in this process, two particularly vulnerable patient groups were chosen. A statistical analysis was performed by using the laboratory information system for checking all ICU laboratory assignments over a 1-month time period under initial conditions and again 1 year after the study. A stay in the ICU of at least 7 days was set as the filter criterion. For the initial 1-month query period, 32 patients met this requirement; for the 1-month query period 1 year after the study, 33 patients met the requirement. All individual laboratory assignments for these patients were checked during each patient's complete ICU stay. The nominal volumes of the corresponding blood tubes, received in the laboratory and electronically confirmed, were recorded. The blood drawn for diagnostic purposes during each patient's complete ICU stay was calculated on this basis, and the initial and final average diagnostic blood loss for this patient group was determined. The same approach was used for geriatric patients' blood withdrawal profiles for a period of 1 week before the tube change ( 86 patients) and for 1 week 1 year after the change (84 patients).

Third, the initial and final number of tubes used hospitalwide and for ICU and geriatric patients per year was determined and the total volume of patients' blood collected was calculated and compared.

\section{Improvement of preanalytics}

Preanalytical processes in different areas of interest were investigated in order to determine preanalytical errors, their potential to reduce

Table 1: Change in nominal tube volumes.

\begin{tabular}{lllr}
\hline Type of tube & Initial tube volume & Final tube volume & Change of volume \\
\hline Blood gas analysis & $2 \mathrm{~mL}$ & $1.6 \mathrm{~mL}$ & $-20 \%$ \\
Heparin plasma & $4.5 \mathrm{~mL}$ & $3 \mathrm{~mL}$ & $-33 \%$ \\
Serum & $7.5 \mathrm{~mL}$ & $6 \mathrm{~mL}$ & $-20 \%$ \\
& & $4 \mathrm{~mL}$ (geriatrics) & $-47 \%$ \\
Citrate plasma & $3 \mathrm{~mL}$ & $2.7 \mathrm{~mL}$ & $-10 \%$ \\
& & $1.8 \mathrm{~mL}$ (geriatrics) & $-40 \%$ \\
Glucose/lactate $(\mathrm{NaF})$ & $2.7 \mathrm{~mL}$ & $2 \mathrm{~mL}$ & $-26 \%$ \\
Sedimentation rate & $3.5 \mathrm{~mL}$ & $1.8 \mathrm{~mL}$ & $-49 \%$ \\
EDTA hematology & $2.7 \mathrm{~mL}$ & $3 \mathrm{~mL}$ & $+10 \%$ \\
EDTA immune hematology & $4.9 \mathrm{~mL}$ & $6 \mathrm{~mL}$ & $+22 \%$ \\
\hline
\end{tabular}


Table 2: Observed aspects of preanalytical phase and risks of non-compliance.

\begin{tabular}{lll}
\hline Observed aspect & Best practice $[4,5]$ & Risk of non-compliance \\
\hline Patient identification & $\begin{array}{l}\text { Minimum three independent identification } \\
\text { criteria with non-leading questions } \\
\text { Label in correct position and orientation }\end{array}$ & $\begin{array}{l}\text { Patient misidentification } \\
\text { Sample labeling }\end{array}$ \\
$\begin{array}{lll}\text { Underfilling of tubes } \\
\text { All tubes should be correctly filled to ensure } \\
\text { correct additive to blood ratio }\end{array}$ & $\begin{array}{l}\text { Insufficient sample volume for tests requested } \\
\text { Citrate: }<90 \% \text { falsely prolonged clotting times }\end{array}$ \\
& & $\begin{array}{l}\text { EDTA: }<75 \% \text { end concentration higher than recommended, impact on } \\
\text { cell morphology } \\
\text { Heparin: specific assays may give erroneous results, e.g. Troponin I }\end{array}$ \\
\hline
\end{tabular}

blood sample quality and therefore to lead to unnecessary repeated blood collections which, in turn, leads to a delay in obtaining analytical results. In total, 54 blood collections in six representative hospital departments, including the emergency room (ER), were observed by trained specialists, using standardized questionnaires (BD Laboratory Consulting Services ${ }^{\circledR}$ ). A total of 563 blood samples were visually inspected in the central laboratory in order to evaluate if they met the criteria for sample acceptance (Table 2) (correct anticoagulant, additive tubes not underfilled, etc.).

The observed preanalytical errors were used as a base for developing hospitalwide training measures. Two kick-off events for a wide range of medical staff were performed in order to raise awareness of the impact of preanalytical errors on laboratory diagnostics and to gain acceptance for the forthcoming training. The staff received specific training on the processes associated with the preanalytical errors observed [4, 5]. After the change of blood collection equipment, an observation period of approximately 3 months was used to identify departments which needed additional training. After 1 year, a re-audit was performed, reviewing the same aspects as in the initial audit for 48 blood collections and 534 blood samples.

\section{Results}

\section{Quantification of the blood volume drawn}

As the focus of this study was a reduction in diagnostic blood loss, a detailed evaluation was performed for two different clinical departments, both caring for patients especially prone to the development of iatrogenic anemia. For geriatric patients, particularly vulnerable due to their multimorbidity, diagnostic blood loss during 1 week was nearly halved (Table 3).
Intensive care patients are another patient group that is prone to the development of iatrogenic anemia [6-8]. In particular, patients with a longer stay in the ICU have to undergo blood tests several times a day for monitoring purposes. In this context, blood sampling for point-of-care testing (POCT) diagnostics, such as blood gas analysis (BGA), must also be considered when determining overall diagnostic blood loss.

The mean diagnostic blood loss for this group was reduced by $27.2 \%$ per week (Table 4 ). The fact that savings in the ICU were smaller than in geriatric patients may be partially due to the high usage of POCT BGA, which also gives information on additional parameters such as electrolytes and hemoglobin as part of the same analysis.

An estimation of diagnostic blood loss, for the entire hospital as well as for the ICU and geriatrics, was performed by comparing the total blood volume initially collected over 1 year, as measured by the number and volumes of tubes used, with that 1 year after the changes had been made. This approach is an approximation as not all of the tubes collected necessarily enter the diagnostic process. Tubes can be discarded for a number of reasons, such as the use of blood collection tubes in training courses, blood collection failures, etc. These discarded tubes cannot be excluded in this model, however they affect both calculation periods in the same manner. A correlation with hospital patient numbers per year revealed a significant saving in the number of blood collection tubes per patient used per year (Table 5). The total increase of $1 \%$ in tubes per year after the change of blood collection equipment was paralleled by a $4.9 \%$ increase in patient numbers and

Table 3: Mean diagnostic blood loss for geriatric patients.

\begin{tabular}{llll}
\hline Mean diagnostic blood loss & Initial $(\mathrm{n}=\mathbf{8 6}$ patients $)$ & Final $(\mathrm{n}=\mathbf{8 4}$ patients $)$ & \\
\hline Derived from detailed analysis of & $3.95 \mathrm{~mL} /$ patient/day & $2.35 \mathrm{~mL} /$ patient/day & \\
patients over a 1-month period & $27.65 \mathrm{~mL} /$ patient/week & $16.45 \mathrm{~mL} /$ patient/week & $-40.4 \%$ \\
\hline
\end{tabular}


Table 4: Mean diagnostic blood loss per week for ICU patients.

\begin{tabular}{|c|c|c|c|c|c|c|c|c|}
\hline ICU patients $n=32$ & $\begin{array}{l}\text { Serum } \\
7.5 \mathrm{~mL}\end{array}$ & $\begin{array}{r}\text { Lithium heparin } \\
4.5 \mathrm{~mL}\end{array}$ & $\begin{array}{r}\text { EDTA } \\
2.7 \mathrm{~mL}\end{array}$ & $\begin{array}{r}\text { EDTA } \\
4.9 \mathrm{~mL}\end{array}$ & $\begin{array}{r}\text { Citrate } \\
3 \mathrm{~mL}\end{array}$ & $\begin{array}{l}\text { BGA } \\
2 \mathrm{~mL}\end{array}$ & $\begin{array}{r}\text { ESR } \\
3.5 \mathrm{~mL}\end{array}$ & $\begin{array}{r}\text { Total } \\
(\mathrm{mL} / \text { patient/week) }\end{array}$ \\
\hline \multicolumn{9}{|l|}{ Initial } \\
\hline Number of tubes per patient & 0.660 & 5.351 & 6.364 & 0.739 & 7.186 & 19.020 & 0.027 & \\
\hline Blood volume, $\mathrm{mL}$ & 4.950 & 24.080 & 17.182 & 3.621 & 21.558 & 38.040 & 0.095 & 109.52 \\
\hline ICU patients $n=33$ & $\begin{array}{r}\text { Serum } \\
6 \mathrm{~mL}\end{array}$ & $\begin{array}{r}\text { Lithium heparin } \\
3 \mathrm{~mL}\end{array}$ & $\begin{array}{l}\text { EDTA } \\
3 \mathrm{~mL}\end{array}$ & $\begin{array}{l}\text { EDTA } \\
6 \mathrm{~mL}\end{array}$ & $\begin{array}{l}\text { Citrate } \\
2.7 \mathrm{~mL}\end{array}$ & $\begin{array}{r}\text { BGA } \\
1.6 \mathrm{~mL}\end{array}$ & $\begin{array}{r}\text { ESR } \\
1.8 \mathrm{~mL}\end{array}$ & $\begin{array}{r}\text { Total } \\
(\mathrm{mL} / \text { patient/week) }\end{array}$ \\
\hline \multicolumn{9}{|l|}{ Final } \\
\hline Number of tubes per patient & 0.424 & 5.136 & 5.052 & 0.611 & 5.527 & 17.472 & 0.023 & \\
\hline Blood volume, $\mathrm{mL}$ & 2.544 & 15.408 & 15.156 & 3.666 & 14.923 & 27.955 & 0.041 & $79.70(-27.2 \%)$ \\
\hline
\end{tabular}

ESR, erythrocyte sedimentation rate.

Table 5: Total number of tubes used for blood collection within the hospital and total diagnostic blood volume per year.

\begin{tabular}{|c|c|c|c|}
\hline Tube type & $\begin{array}{l}\text { Initial tube number per } \\
\text { year }\end{array}$ & Final tube number per year & $\begin{array}{l}\text { Overall tube number } \\
\text { per patient per day }\end{array}$ \\
\hline Serum & $56500(7.5 \mathrm{~mL}$ tube $)$ & $\begin{array}{l}46200 \text { (6 mL tube) } \\
4200 \text { (4 mL tube) }\end{array}$ & \\
\hline Lithium heparin & 33950 (4.5 mL tube) & 33000 (3 mL tube) & \\
\hline EDTA & $\begin{array}{l}62100(2.7 \mathrm{~mL} \text { tube }) \\
11700 \text { (4.9 mL tube) }\end{array}$ & $\begin{array}{l}40300 \text { (3 mL tube) } \\
21900 \text { ( } 2 \mathrm{~mL} \text { tube) } \\
16800 \text { (6 mL tube) }\end{array}$ & $-13 \%$ \\
\hline Citrate & 47400 (3 mL tube) & $\begin{array}{l}33100(2.7 \mathrm{~mL} \text { tube }) \\
16800(1.8 \mathrm{~mL})\end{array}$ & \\
\hline ESR & 1450 (3.5 mL tube) & 4300 (1.8 mL tube) & \\
\hline Blood gas analysis & 29100 (2 mL tube) & 27560 (1.6 mL filling volume of blood) & \\
\hline Glucose/lactate & 1450 (2.7 mL tube) & 2200 (2 mL tube) & \\
\hline Total blood volume per year & $1011 \mathrm{~L}$ & $834 \mathrm{~L}(-17.5 \%)$ & \\
\hline $\begin{array}{l}\text { Reduction in the use of blood tubes } \\
\text { for ICU and geriatric patients per year }\end{array}$ & Initial & Final & \\
\hline $\begin{array}{l}\text { ICU patients } \\
\text { Geriatric patients }\end{array}$ & $\begin{array}{l}2.11 \text { tubes/patient/day } \\
2.13 \text { tubes/patient/day }\end{array}$ & $\begin{array}{l}2.10 \text { tubes/patient/day } \\
2.03 \text { tubes/patient/day }\end{array}$ & $\begin{array}{l}-0.5 \% \\
-4.7 \%\end{array}$ \\
\hline
\end{tabular}

ESR, erythrocyte sedimentation rate.

thereby constituted a reduction in the number of tubes per patient per day. In addition, nominal tube volumes were reduced. The total blood volume saved by means of the reduction of blood collection tube volumes was averaged at approximately $177 \mathrm{~L}$ per year. This corresponds to a decrease in the blood drawn for diagnostic purposes of $17.5 \%$ for all patients in the hospital. Also, the number of tubes per day and per patient were significantly reduced by $13 \%$ (Table 5). For ICU patients, tubes per patient and per day decreased slightly by $0.5 \%$; for geriatric patients, the decrease was $4.5 \%$ (Table 5).

Reduction of the overall volume of blood collected can be converted into erythrocyte concentrate equivalents [9] for the 384-bed hospital. The average saving of $177 \mathrm{~L}$ blood corresponds to approximately 805 erythrocyte concentrate equivalents per year $(-37 \%)$. The transfusion of erythrocyte concentrates during the 1-month time interval analyzed was reduced for ICU patients by 7.5\%. For the comparison of the two geriatric patient groups, a slight increase from 0 to 3 erythrocyte concentrates was observed (Table 6).

\section{Preanalytics on the ward}

Correct identification of patients is an important aspect in avoiding unnecessary repeated blood collections. The omission of adequate patient identification carries a high risk of patient misidentification and therefore potential 
Table 6: Transfusion of erythrocyte concentrates during the 1-month time interval analyzed.

\begin{tabular}{llll}
\hline & Initial & Final & \\
\hline ICU patients & 81 erythrocyte concentrates & 75 erythrocyte concentrates & $-7.5 \%$ \\
Geriatric patients & 0 erythrocyte concentrates & 3 erythrocyte concentrates & n.d \\
\hline
\end{tabular}

Table 7: Improvement of preanalytics as observed in initially 54, finally 48 blood collections on the ward and in initially 563 , finally 534 sample inspections in the laboratory.

\begin{tabular}{lll}
\hline Patient identification & Initial $(\mathrm{n}=54$ blood collections) & Final ( $\mathrm{n}=48$ blood collections) \\
Application of three independent patient ID criteria & $17 \%$ & $63 \%$ \\
Blood collection tube & Initial $(\mathrm{n}=563)$ & Final $(\mathrm{n}=534)$ \\
Correct tube labeling & $33 \%$ & $67 \%$ \\
Correct filling volume & Initial $(\mathrm{n}=563)$ & Final $(\mathrm{n}=534)$ \\
Blood coagulation analysis (citrate) (>90\%) & $97 \%$ & $95 \%$ \\
Hematology (>75\%) & $89 \%$ & $85 \%$ \\
Clinical chemistry (serum) (>75\%) & $77 \%$ & $80 \%$ \\
Clinical chemistry (lithium heparin) $(>75 \%)$ & $31 \%$ & $80 \%$ \\
\hline
\end{tabular}

patient harm. In this study, it was determined whether three independent patient identification (ID) criteria were correctly checked in the identification process. Compared with the initial situation, training led to a significant improvement (3.7-fold) in patient identification using three independent ID criteria (Table 7).

Additionally, selection of the correct blood collection tube for the analysis to be performed and the correct labeling of the tubes with patient data, typically by applying barcode labels, are essential. The following non-compliances with respect to blood tube labeling, leading to a delay in laboratory analysis as these tubes require additional manual processing or cause tube rejection in the laboratory, were recorded: label was glued at an oblique angle or in a position which did not follow manufacturer's recommendations, label stood out or label indicated a different additive to the actual tube type. After training and clarification of the importance of a correct labeling and tube choice for laboratory TAT, the initial rate of correct tube labeling almost doubled in the re-audit (Table 7).

\section{Improvement of efficiency in the central laboratory}

Initially, 563 blood collection tubes of different sample types were checked for sample quality and observed from their arrival in the laboratory until they were loaded into the appropriate analyzer. One year after the change in the blood collection equipment and training, 534 tubes were checked. Sample rejection rates have not been included in this study due to limited laboratory information system access.

As shown in Table 7, the initial rate of citrate tubes filled below $90 \%$ was already low. This had been achieved by continuous training and feedback using the hospital's quality management system. Underfilling rates of citrate tubes 1 year after the changes had slightly increased. The rate of EDTA tubes filled below $75 \%$ also increased slightly. Further continuous training is a mandatory part of the quality management system. The rate of clinical chemistry analysis serum tubes filled below $75 \%$ was reduced slightly. In the case of lithium heparin tubes, the change was significant. The number of tubes filled more than $75 \%$ increased from $31 \%$ to $80 \%$ (Table 7 ).

\section{Discussion}

In the present study, a multimodal concept was established in order to reduce diagnostic blood loss and to improve laboratory performance in terms of preanalytical quality and TAT.

This targeted approach reviewed the initial situation to identify optimization potential and then implemented a multistep improvement strategy to enable higher efficiency and thereby improve patient care.

In this study, a reduction of diagnostic blood loss was achieved by reducing blood collection tube volumes. The effect of this measure was evaluated for geriatric as well as ICU patients as both of these are 
especially vulnerable to developing iatrogenic anemia due to diagnostic blood loss.

Additionally, an estimation of success was performed for the entire hospital. As this study was conceived as a first attempt in quantifying blood savings by the measures described above, the impact on patient groups other than geriatric and ICU could not be investigated in detail. Due to the relatively small groups included in the study, the annual rise in patient numbers within the hospital and the new integration of a vascular surgery unit into the hospital within the time period analyzed, an exact correlation of the saved erythrocyte concentrate equivalents with a saving in transfused erythrocyte concentrates could not be performed. A slight decrease in transfusion rates at the ICU $(7.5 \%)$ was observed between the two 1-month periods compared. In the geriatric ward, an increase was observed from 0 to 3 erythrocyte concentrates. In order to gain more reliable data, larger study groups and longer analysis intervals are required as the medical backgrounds of the ICU patients and geriatric patients were highly heterogeneous, thereby resulting in varying transfusion probabilities over the two time periods analyzed.

An analysis of blood-saving possibilities in ICU patients showed that POCT BGA, giving information on a variety clinical chemistry and hematological parameters besides blood gases from a single analysis, plays an important role in diagnostic blood loss for this patient group, as recently also shown by Hintereder [9]. POCT diagnostics are performed in parallel with central laboratory diagnostics, limiting the potential saving of blood by the approach used here. An additional issue is that the easy accessibility and range of analyses possible with modern POCT in the ICU or the ER can lead to an increase in the frequency of blood analysis which therefore limits the effects of blood-saving strategies.

This shows, even though POCT has undeniable benefits, interactions of POCT with central laboratory diagnostics and an overall analysis process always need to be carefully considered. For this reason, a reasonable laboratory TAT is important to minimize non-critical use of POCT diagnostics. TAT is one of the most important and measurable characteristics for laboratories and for hospital benchmark purposes. It is also viewed by physicians and nurses as one of the most important measures for the service quality of the laboratory [10, 11]. In particular, faulty preanalytical processes make a large contribution to a prolonged TAT.

In order to improve laboratory TAT by reducing the frequency of preanalytical errors, specific training measures were carried out. Best practice preanalytical processes are key for obtaining high-quality laboratory diagnostics.
Regular training and close supervision of the blood-collecting medical staff help to increase awareness of the relevance of preanalytics, such as inadequate filling as observed in this study. Inadequate filling of tubes is one of the main reasons for repeated blood collections. Underfilling, especially as initially observed in heparin tubes, may be due to emergency situations and subsequent time pressure during blood collections. Even if the sample volume was sufficient for the test requested, sample quality may be compromised because of an increased concentration of the additive (Table 2).

The optimization of processes as described in this study is an important building block in PBM. In addition to favorable effects for the individual patient, it could also reduce the need for red blood cell transfusions and thus increase blood bank stocks to ensure that those patients who really need blood transfusions will receive them in the future.

Both, improving preanalytical processes and reducing nominal tube collection volumes, serve as a lab-side module of the PBM for the prevention of hospital acquired anemia by responsible handling of patients' blood resources.

As blood drawn for laboratory analysis, intervention and surgeries form a large proportion of the reasons for hospital-acquired anemia [12], blood volumes required for laboratory diagnostics need to be considered in this context. The more severely ill the patients are, the more intensive the laboratory diagnostics become and the more blood will be needed for diagnostic purposes. Additionally, the efficiency of erythropoiesis is limited in seriously ill patients. As a result of these two factors, the risk of development of iatrogenic anemia in this patient group is disproportionately increased.

This can be avoided by implementation of different strategies, such as reducing diagnostic blood loss, minimizing perisurgical blood loss and using evidence-based coagulation and hemotherapy concepts with guidelineappropriate indications for blood product administrations. The impact of structured PBM in German hospitals has been the subject of multicentric studies. Thus, in addition to unambiguous short- and medium-term benefits for patients, a total cost saving for hospitals has been shown [13-18]. Fischer et al. [19, 20] indicated the importance of reduced blood collection in 2014.

The multimodal approach initiated by laboratory diagnostics as described here can be interpreted as a valuable lab-side contribution to PBM, whose effectiveness is demonstrated in this study. Considering PBM as a continuous optimization process, a possible next step for further volume reduction could be the implementation of computer-based laboratory request control using 
strict indication-related laboratory profiles based on established laboratory diagnostic pathways [21].

Author contributions: All authors have accepted responsibility for the entire content of this submitted manuscript and approved submission.

Research funding: Janine Spethmann and Kathrin Schlatterer received congress fee and travel expense reimbursements by BD Life Sciences.

Employment or leadership: Kathrin Schlüter is employed by BD Life Sciences/Preanalytical Systems, Heidelberg, Germany.

Honorarium: None declared.

Competing interests: The funding organization(s) played no role in the study design; in the collection, analysis, and interpretation of data; in the writing of the report; or in the decision to submit the report for publication.

\section{References}

1. World Health Organization. Global Forum for Blood Safety: Patient Blood Management. 2011; http://www.who.int/bloodsafety/events/gfbs_01_pbm_concept_paper.pdf

2. Ranasinghe T, Freeman WD. "ICU vampirism” - time for judicious blood draws in critically ill patients. Br J Haematol 2014;164:302-3.

3. Levi M. Twenty-five million litres of blood into the sewer. J Thromb Haemost 2014;12:1592.

4. Clinical and Laboratory Standards Institute. Procedures for the collection of diagnostic blood specimens by venipuncture; Approved Standard; PA: CLSI $6^{\text {th }}$ ed., Wayne 2007:H3-A6.

5. World Health Organization. WHO guidelines on drawing blood: best practices in phlebotomy, WHO Document Production Services 2010, Geneva.

6. Wisser D, van Ackern K, Knoll E, Wisser H, Bertsch T. Blood loss from laboratory tests. Clin Chem 2003;49:1651-5.

7. Chant C, Wilson G, Friedrich JO. Anemia, transfusion, and phlebotomy practices in critically ill patients with prolonged ICU length of stay: a cohort study. Crit Care 2006;10:R140.

8. Tosiri P, Kanitsap N, Kanitsap A. Approximate iatrogenic blood loss in medical intensive care patients and the causes of anemia. J Med Assoc Thai 2010;93:271-6.

9. Hintereder G. Patient blood management - Labormedizinische Unterstützung und POCT. J Lab Med 2017;41:219-27.
10. McCall SJ, Souers RJ, Blond B, Massie L. Physician satisfaction with clinical laboratory services: a College of American Pathologists Q-Probes study of 81 institutions. Arch Pathol Lab Med 2016;140:1098-103.

11. Jones BA, Walsh MK, Ruby SG. Hospital nursing satisfaction with clinical laboratory services: a College of American Pathologists Q-Probes study of 162 institutions. Arch Pathol Lab Med 2006;130:1756-61.

12. Quaseem A, Alguire P, Dallas P, Feinberg LE, Fitzgerald FT, Horwitch C, et al. Appropriate use of screening and diagnostic tests for foster high-value, cost-conscious care. Ann Intern Med 2012;156:47-9.

13. Farmer SL, Towler SC, Leahy MF, Hofmann A. Drivers for change: Western Australia Patient Blood Management Program (WA PBMP), World Health Assembly (WHA) and Advisory Committee on Blood Safety and Availability (ACBSA). Best Pract Res Clin Anaesthesiol 2013;27:43-58.

14. Meybohm P, Fischer DP, Geisen C, Müller MM, Weber CF, Herrmann E, et al. Safety and effectiveness of a Patient blood management (PBM) program in surgical patients - the study design for a multi-centre prospective epidemiologic non-inferiority trial. BMC Health Serv Res 2014;14:576.

15. Meybohm P, Herrmann E, Steinbicker AU, Wittmann M, Gruenewald $M$, Fischer $D$, et al. Patient blood management is associated with a substantial reduction of red blood cell utilization and safe for patient's outcome: a prospective, multicenter cohort study with a noninferiority design. Ann Surg 2016;264:203-11.

16. Zacharowski K, Spahn DR. Patient blood management equals patient safety. Best Pract Res Clin Anaesthesiol 2016;30:159-69.

17. Fischer DP, Zacharowski K, Müller MM, Geisen C, Seifried E, Müller $\mathrm{H}$, et al. Patient blood management implementation strategies and their effect on physicians' risk perception, clinical knowledge and perioperative practice - the Frankfurt experience. Transfus Med Hemother 2015;42:91-7.

18. Müller MM, Fischer D, Stock U, Geisen C, Steffen B, Nussbaumer J, et al. Patient blood management - The preoperative patient. Anasthesiol Intensivmed Notfallmed Schmerzther 2014;49: 246-53.

19. Fischer D, Geisen C, Steffen B, Meybohm P, Schmitz-Rixen T. Patient blood management - The inpatient care. Anasthesiol Intensivmed Notfallmed Schmerzther 2014;49:256-64.

20. Fischer DP, Zacharowski K, Meybohm P. Savoring every drop vampire or mosquito? Crit Care 2014;18:306.

21. Hofmann W, Aufenanger J, Hoffmann G (eds.). Laboratory diagnostic pathways: clinical manual of screening methods and stepwise diagnosis. Berlin/Boston: Walter de Gruyter GmbH \& Co KG, 2016. 